\title{
METODOLOGIA DE INSPEÇÃO TERMOGRÁFICA POR CAMERA EM DRONE PARA DETECTAR ENTRADA FALSA DE AR EM DUTOS DE GÁS ENTRE CALDEIRA E PRECIPITADOR ELETROSTÁTICO
}

Marco F. Borges, Ms.Eng. 1; Herman A. Lepikson, Dr.Eng. 2; Ricardo Mariano³; Mauro B. Abram ${ }^{4}$

${ }^{1}$ Programa de Pós-graduação em Engenharia Industrial, UFBA; Salvador/Bahia; marcofabiossa@hotmail.com

2 Programa de Pós-Graduação em Gestão e Tecnologia, Senai Cimatec; Salvador/Bahia

${ }^{3}$ Suzano Papel e Celulose SA; Jacareí /SP

${ }^{4}$ PROFNIT - Programa de Pós-Graduação em Propriedade Intelectual e Transferência de Tecnologia para a Inovação; Salvador/Bahia

Resumo: Um problema grave que ocorre entre caldeira e precipitador termostático é a entrada falsa de ar nos dutos de gases. A proposta deste trabalho foi apresentar e validar uma metodologia de inspeção externa destes dutos, durante operação da caldeira, por câmera termográfica embarcada em drone, para direcionar os devidos reparos durante o shutdown. Pela análise das imagens termográficas captadas, foram identificados 132 não conformidades nos dutos inspecionados, dos quais 20 foram referentes a pontos frios, como potenciais locais de entrada falsa de ar para os gases. Com a utilização da metodologia proposta neste trabalho, ficou bastante evidente os ganhos substanciais quanto a qualidade da informação produzida, reduções de custo e tempo operacional da inspeção e garantia da saúde e vida dos envolvidos.

Palavras-Chave: Inspeção; termografia; Drone; Caldeira; Precipitador Eletrostática.

\section{THERMOGRAPHIC INSPECTION METHODOLOGY BY DRONE- EMBEDDED CAMERA TO DETECT FALSE ENTRY OF AIR INTO THE GAS DUCTS IN BOILER AND ELECTROSTATIC PRECIPITATOR}

\begin{abstract}
A serious problem that occurs between boiler and thermostatic precipitator is the false entry of air into the gas ducts. The purpose of this paper was to present and validate a methodology for external inspection of these pipelines, during boiler operation, by drone-embedded thermographic camera, to direct due repairs during shutdown. Through the analysis of the captured thermographic images were identified 132 issues in the inspected ducts, of which 20 were related to cold spots, as potential false air entry points for the gases. With the methodology proposed in this paper, it was quite evident the substantial gains regarding the quality information produced, cost reductions and operational time of inspection, and guaranteeing the health and life of those involved.
\end{abstract}

Keywords: Inspection; Thermography; UAS; Boiler; Electrostatic Precipitator. 


\section{INTRODUÇÃO}

No Brasil, por volta de $95 \%$ das fábricas de celulose utilizam o processo "Kraft", no qual se obtém a pasta a partir de um meio alcalino a base de sulfeto de sódio (Na2S) e de hidróxido de sódio $(\mathrm{NaOH})$. Este processo tem duas macrounidades, sendo a primeira responsável por se obter a polpa celulósica. $E$ a segunda, na qual faz parte a caldeira de recuperação química que é a de recuperação e utilidades, cujos objetivos são: recuperar e regenerar os agentes químicos para se obter a polpa ( $\mathrm{Na2S}$ e $\mathrm{NaOH}$ ); fornecer água, vapor, energia elétrica e ar comprimido para os processos em geral; e tratar os efluentes [1].

\subsection{O Problema}

Os gases de combustão da caldeira são conduzidos por dutos com isolante térmico, a pressão negativa, até o ventilador centrífugo, que introduz os gases no precipitador eletrostático, através de eletrodos e placas coletoras, os particulados em suspensão nos gases, são ionizados e capturados pelo precipitador, normalmente em torno de $99,9 \%$ das partículas. Envia desta forma, gás limpo para a atmosfera, atendendo as legislações ambientais [1].

Um problema grave que ocorre neste processo é a entrada falsa de ar (atmosférico) nos dutos de captação dos gases de combustão, oriundos da caldeira de recuperação química para o precipitador eletrostático, devido a descontinuidades nestes dutos. A redução de temperatura dos gases de entrada pelo aumento de umidade, possibilita a ocorrência de corrosão no precipitador, do ponto de vista mecânico [2]. "Em gases com temperatura abaixo de $176^{\circ} \mathrm{C}$, a umidade presente é absorvida pela superfície da partícula de pó, o que pode reduzir a resistividade do pó" [2], podendo afetar a máxima eficiência do precipitador. Portanto, faz-se necessário a realização de inspeção visual na chapa externa do duto, normalmente de alumínio rebitado, para identificar e eliminar as entradas falsas de ar. Na figura 1 podemos verificar dados do objeto deste estudo de caso.

Figura 1. Detalhes construtivos do duto de captação do Precipitador Termostático
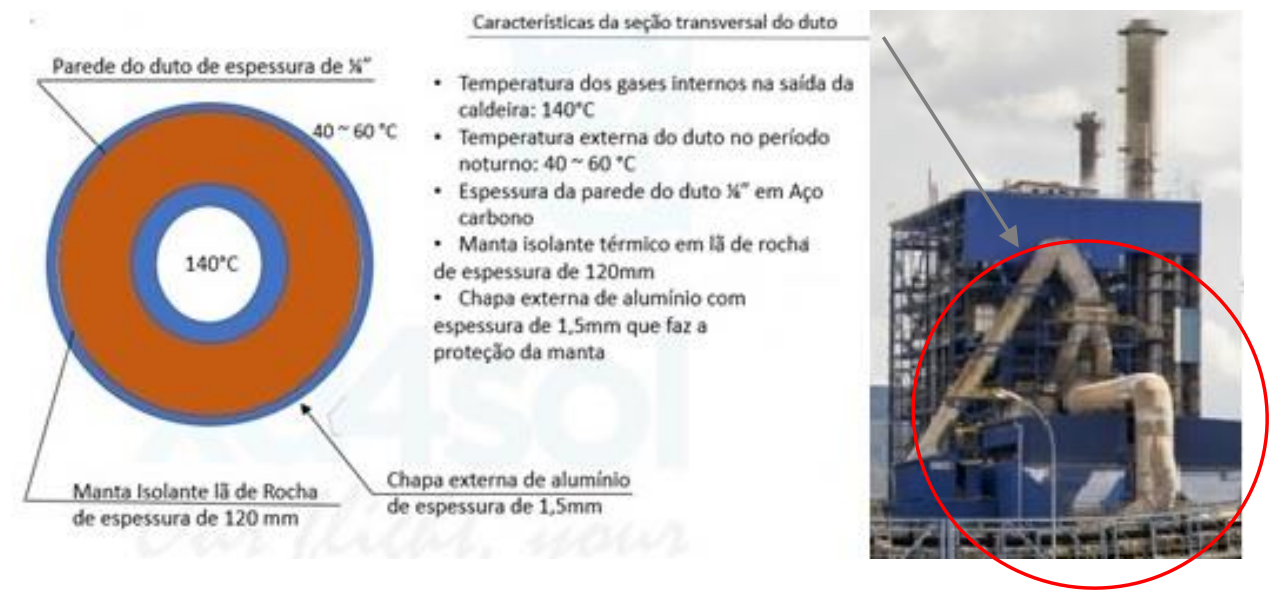


\subsection{Objetivo}

A proposta deste trabalho foi apresentar e validar uma metodologia de inspeção externa de dutos de captação e exaustão de gases de combustão, entre uma caldeira de recuperação química o precipitador termostático e a chaminé, durante sua operação, por câmera termográfica embarcada em drone.

O objetivo foi a detecção de pontos de menor temperatura na superfície das chapas estruturais de alumínio, indicando possíveis pontos de entrada falsa de ar para o interior dos dutos de gases, através de descontinuidades. Com a identificação destes pontos, foram direcionados os reparos a serem executados durante 0 shutdown da fábrica, reparos estes que visam proporcionar condições operacionais otimizadas, para que o precipitador termostático opere próximo a sua máxima eficiência, além da redução de condições que favorecem a ocorrência de processos corrosivos.

Diante de uma operação mais eficiente do precipitador termostático temos um ganho no controle de emissões de gases para atmosfera, contribuindo para uma operação ambientalmente mais sustentável. Os ganhos econômicos esperados foram a redução do custo operacional do processo de inspeção com redução de tempo e recursos, sem necessidade de parada do equipamento, que resultou em informação de maior qualidade e mais sistematizada que os processos tradicionais.

Através do direcionamento sistematizado dos reparos destes dutos, a expectativa foi uma potencial redução de custo e tempo na execução dos reparos. Por fim, e não menos importante, conseguiu-se uma redução significativa de exposição humana aos riscos do trabalho em espaços confinados e em altura, atendendo a normas reguladoras NR33 e NR35 do Ministério do Trabalho.

\section{METODOLOGIA}

A metodologia adotada neste trabalho foi uma pesquisa aplicada de um estudo de caso transversal de uma inspeção termográfica, através de câmera embarcada em drone em uma caldeira de recuperação química, na fábrica de celulose da Suzano, localizada em Jacareí, no estado de São Paulo. Esta inspeção ocorreu no período de 31 de julho a 01 de agosto de 2019.

\subsection{Equipamentos utilizados}

$\mathrm{Na}$ execução deste trabalho foram utilizados dois drones de diferentes configurações, mostrados na figura 2, sendo um voltado para inspeções externas em altura e outro especialista em inspeções em espaços confinados. O primeiro foi o Falcon 8 Trinity da Intel, muito utilizado em inspeções offshore, devido sua tripla redundância nos subsistemas essenciais ao voo, conferindo alta confiabilidade e robustez. Foi embarcado uma câmera termal FLIR TAU 2640 radiométrica, de 


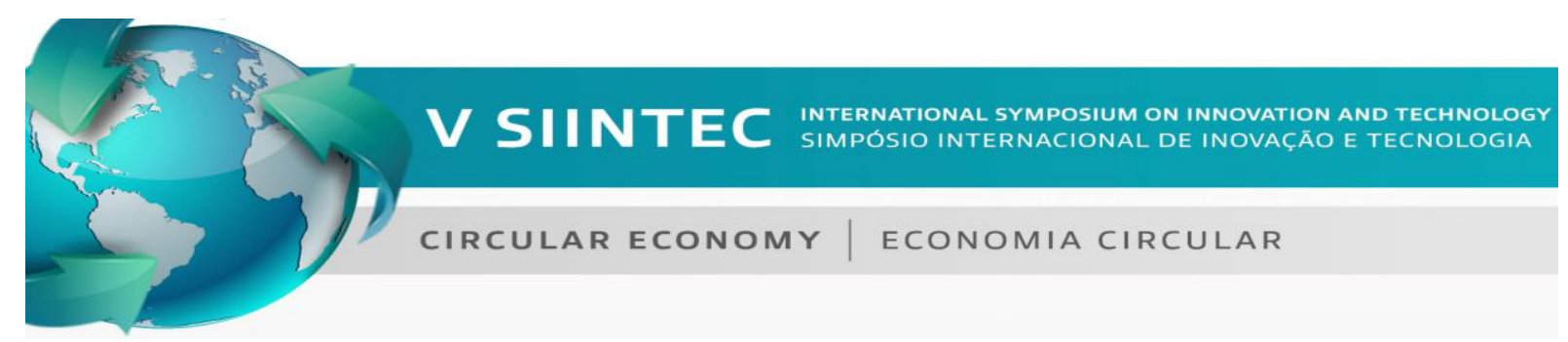

resolução de $640 \times 512$ pixel, comprimento focal de $19 \mathrm{~mm}$ e abertura f/1,25, montada em conjunto com uma filmadora Sony CX410 com zoom de 55x.

O segundo equipamento utilizado foi o Elios, especializado em inspeções em espaços confinados, por ter uma gaiola protetora feita em fibra de carbono, que permite contato e colisão com objetos e iluminação própria por LEDs. São embarcadas neste equipamento duas câmeras, sendo uma câmera RGB Full HD com resolução de até $0,2 \mathrm{~mm}$ o pixel e uma câmera termal FLIR não radiométrica de $160 \times 120$ pixels.

Figura 2. Imagem do drones utilizados embarcados com as câmeras termais: Falcon 8 a esquerda e o Elios a direita
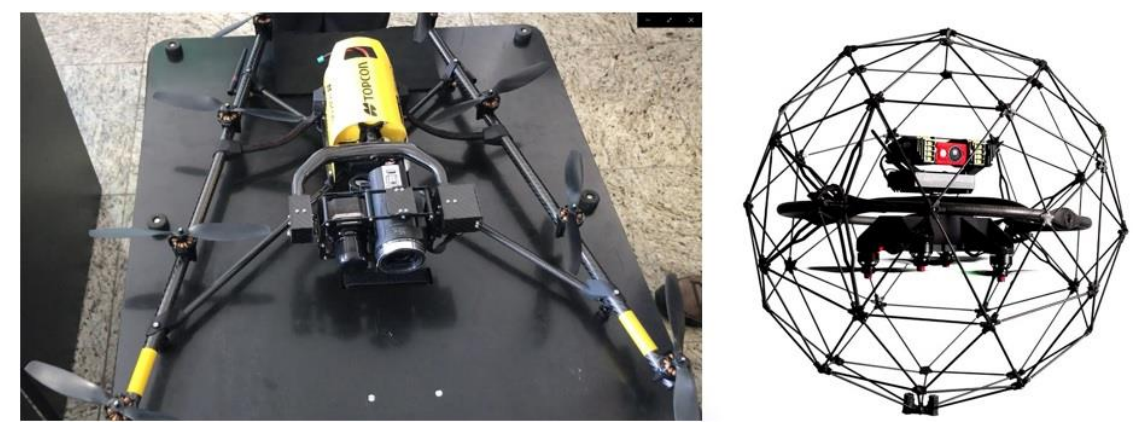

\section{RESULTADOS E DISCUSSÃO}

\subsection{Estado da Arte}

Embora termografia e drones sejam assuntos bastante conhecidos, existem poucos artigos que os considerem juntos, desenvolver trabalhos combinando estas duas tecnologias obtém-se novos benefícios agregados. Uma questão importante a ser considerada neste trabalho é que, tecnicamente, não é uma tarefa fácil garantir que os parâmetros relacionados ao material (como emissividade, cor, diferença de temperatura e reflexão térmica) não influenciem de forma não intencional nas medições termográficas [3].

Figura 3. a) Ausência de isolamento térmico

b) Infiltração de ar. Fonte: Taylor (2013)
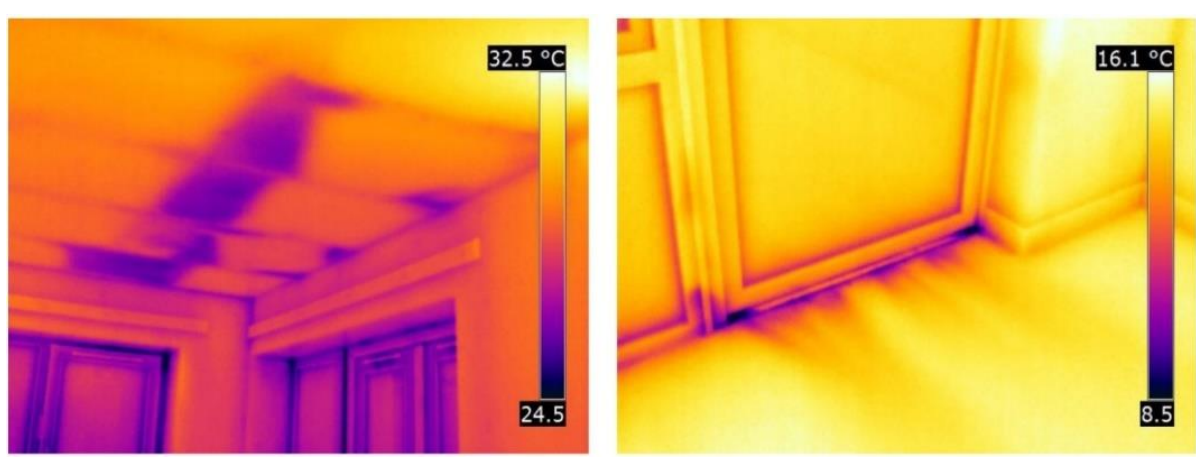
Em termos de projeto detalhado e fabricação, a camada de isolamento precisa ser continua e uniforme para evitar pontes térmicas e a camada de estanqueidade precisa ser continua para reduzir a infiltração [4]. Na figura 3 acima, podemos ver o comportamento térmico resultante da ausência de isolamento térmico e infiltração de ar em um ambiente interno aquecido [4].

Em inspeções de infiltração de ar frio em edificações, o fluxo de ar através dos componentes das edificações causa um gradiente de temperatura em suas superfícies. Sua magnitude depende da natureza e tamanho do ponto de infiltração, o diferencial de pressão $(\Delta \mathrm{P})$ do envelope da edificação e o $\Delta \mathrm{T}$. Inspeções qualitativas termográficas de infravermelho pode ser usado para detectar infiltração de ar em edifícios graças ao gradiente de temperatura. Para uma identificação de vazamento bem-sucedida o procedimento padrão europeu BS EM 13187:1999 de Performance Térmica de Edificações, requer pelo menos um $\Delta \mathrm{T}$ entre as temperaturas interna $\mathrm{e}$ externa de $5^{\circ} \mathrm{C}$ e variação da temperatura externa de $+/-10^{\circ} \mathrm{C}$ nas últimas 24 horas, além da ausência de radiação solar direta na superfície a ser inspecionada por 12 horas [5]. Apesar dos artigos acima tratarem de avaliação de eficiência térmica em edificações, podemos constatar algumas correlações com o processo analisado neste artigo, trazendo argumentos científicos para a metodologia aqui proposta.

Algumas recomendações importantes para a realização de inspeções em infravermelho. Em primeiro lugar, é preciso considerar o efeito da irradiação solar na superfície a ser inspecionada, quando se quer identificar pontes térmicas, preferencialmente a inspeção deve ser realizada antes do nascer do sol. Em segundo lugar, a distância entre o objeto de interesse e a câmera térmica deve ser observada e levada em consideração na interpretação das imagens termais. Por fim, as características da câmera também devem ser consideradas, a saber, as do campo de visão da lente e de resolução da câmera, a resolução mínima utilizada deve ser de $320 \times 240$ pixels. Já uma resolução recomendada para maiores distâncias deve ser de $640 \times 480$ pixels [6]. Outros fatores importantes a serem considerados são a condutividade térmica e emissividade dos materiais inspecionados, reflexão e temperaturas internas e externas [6].

Conforme sumário realizado por Doshvarpassand [7], em uma revisão de artigos publicados de 2013 a 2019 sobre aplicações termográficas mais comuns nas indústrias, que dizem respeito a detecção de vazamento e infiltração, foram apenas $0,5 \%$ dentre todos os segmentos de indústrias pesquisadas, e menos de $2 \%$ dentre aplicações em estruturas e materiais metálicos. Muito pouco na literatura é mencionado sobre aplicação de mecanismos de resfriamento em vez de aquecimento, considerando que os mecanismos de transferência de calor para aquecimento e resfriamento são similares. Isto indica que vale a pena investigar mais sobre os métodos de resfriamento em aplicações de inspeções em campo [7]. 


\subsection{Resultados}

As inspeções externas com o drone Falcon 8 foram realizadas no dia 01 de agosto de 2019 em torno das 06:30 horas da manhã, condições climáticas com pouca neblina, quase nenhum vento e temperatura ambiente em torno dos $15^{\circ} \mathrm{C}$. Também foram realizadas algumas capturas de imagem no dia anterior por volta das 18:00h para fins de comparativos de temperatura entre o final do dia e a manhã. Já as inspeções nos dutos internos com o Elios, foram realizadas por volta das 09:00 horas do mesmo dia, 01 de agosto. Foram necessários apenas 2 profissionais neste trabalho, sendo um piloto e um engenheiro inspetor de qualidade e o tempo total de de 6 horas, para a realização de todo o trabalho de inspeção, através da captação de imagens termográficas e no espectro visível (RGB). Após a realização da inspeção no campo, foram necessários em torno de 4 dias (32 horas) de trabalho de um engenheiro industrial mecânico, em escritório, analisando as imagens e preparando o relatório técnico com as devidas observações, análises e recomendações.

Conforme pode ser observado na tabela 1 , foram encontrados 20 pontos frios, dos quais 17 pontos foram nos dutos de entrada do Precipitador da CR4, como potenciais acessos de entrada de ar frio úmido para dentro dos dutos, se misturando com os gases. Também foram encontrados 37 pontos quentes nas juntas dos dutos e bocas de visita, indicando possíveis descontinuidades, por onde podem também ocorrer entrada falsa de ar. Quando isto acontece antes do Precipitador Termostático, em reação com componentes químicos presentes nos gases, favorece a formação de ácido, que ataca através de processos corrosivos, as paredes das câmaras do Precipitador.

Tabela 1. Sumário das Não Conformidades encontradas na Inspeção dos Dutos

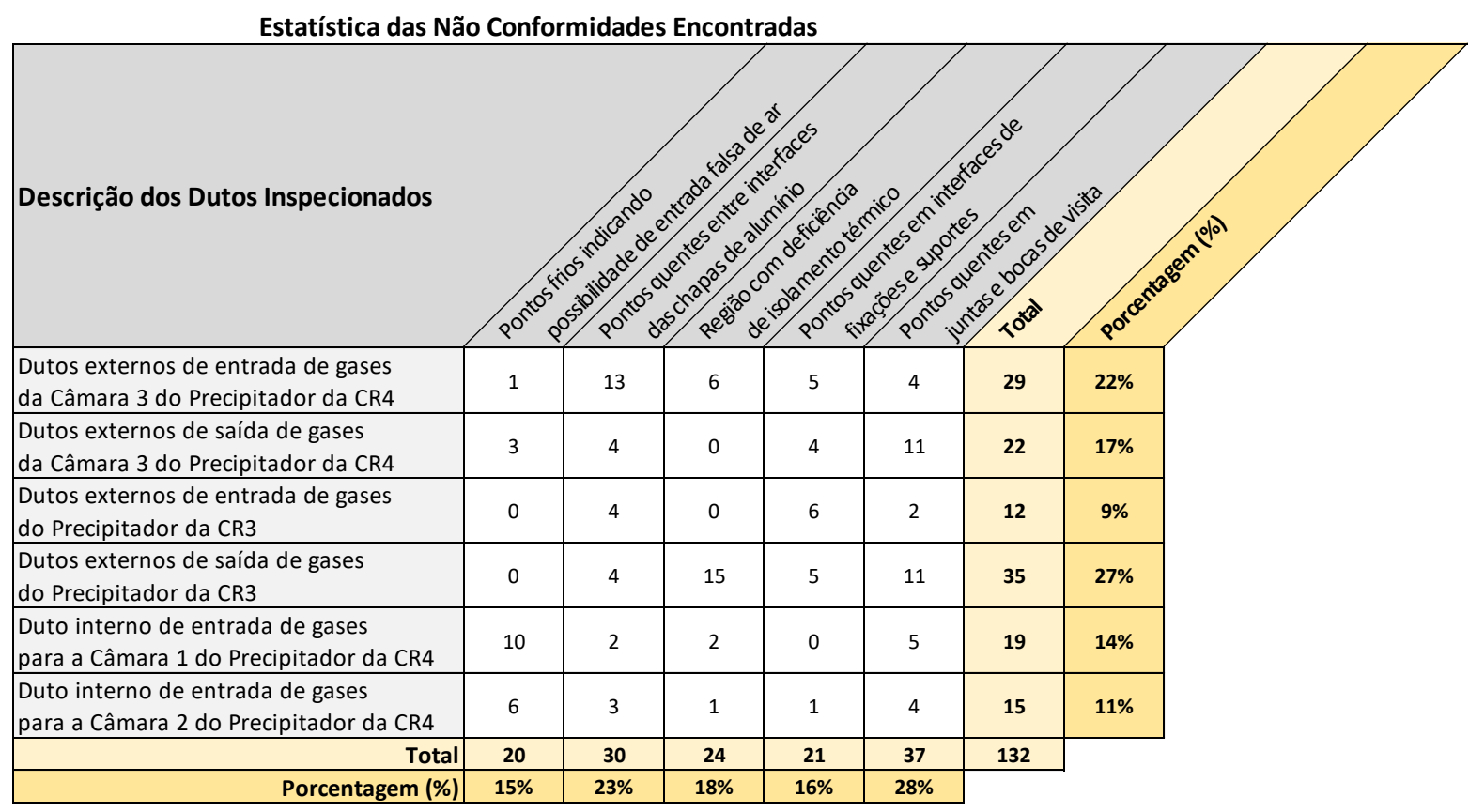


O número de pontos quentes entre interfaces das chapas externas de alumínio encontrados foram um total de 30 pontos, além de 21 pontos quentes em interfaces de fixação com suportes e olhais de transporte. Estes pontos favorecem a entrada de ar úmido para a região da manta térmica, ocasionando processo de oxidação destas mantas, o que faz com elas percam sua eficiência como isolante térmico.

As 24 regiões de baixa eficiência do isolante térmico, permitem pontes térmicas, o que favorece com que os gases tenham significativa queda de temperatura nos períodos da madrugada, isto ocasiona a retenção de sais minerais nas paredes internas dos dutos de gases, reduzindo a seção transversal por onde eles passam. Estas regiões com sais minerais ocasionam pontos quentes internos que favorecem a ocorrência de processos corrosivos, podendo vir a produzir furos nos dutos de passagem dos gases.

Figura 4. Não conformidades encontradas.
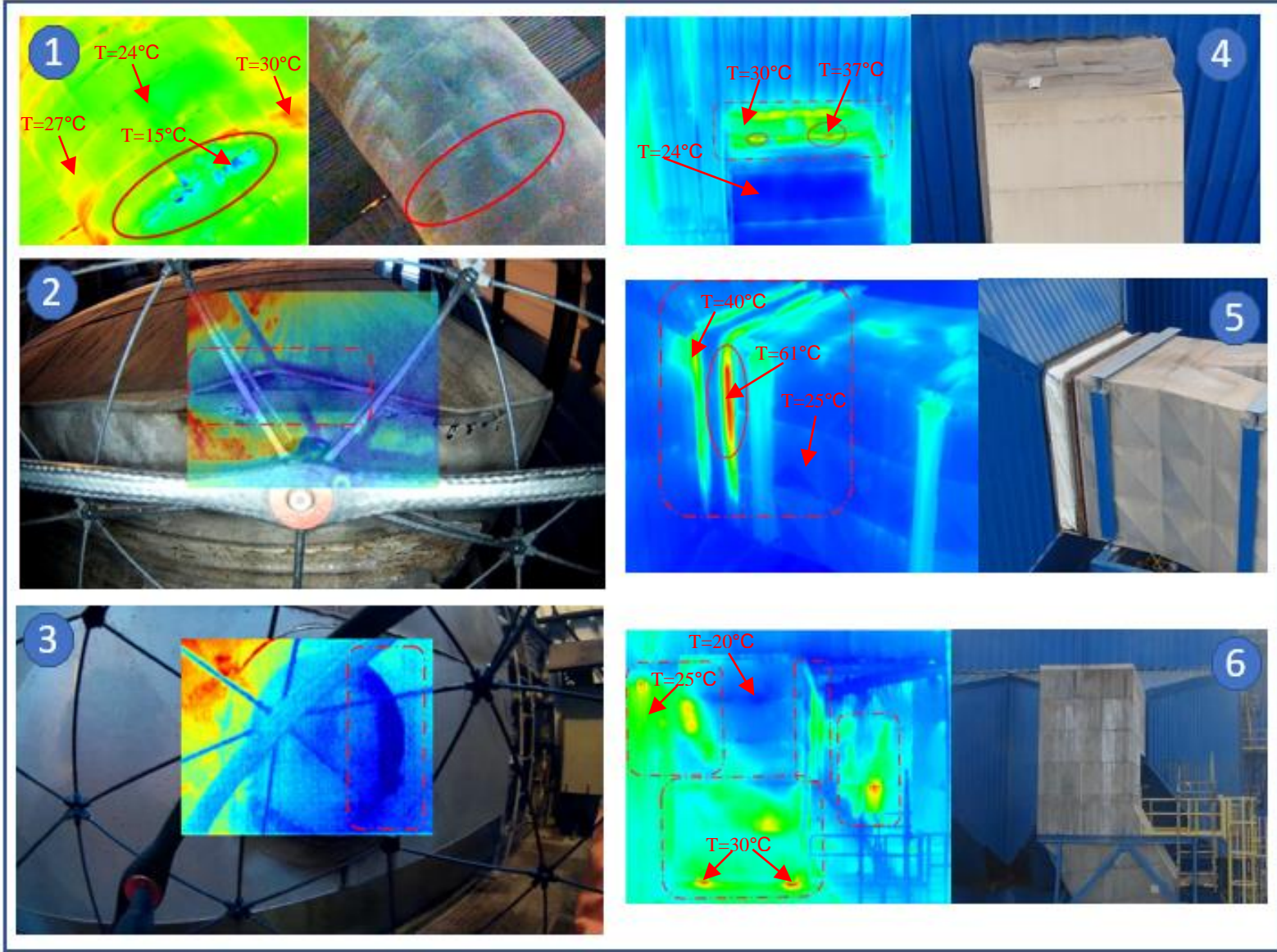

$\mathrm{Na}$ figura 4 acima, podem ser vistos 06 casos das 132 não conformidades encontradas de maior criticidade. $\mathrm{Na}$ imagem 1 encontram-se pontos frios identificados com temperatura de $15^{\circ} \mathrm{C}$ em locais com as chapas bastante deformadas, enquanto as superfícies vizinhas estão com temperatura em torno de $24^{\circ} \mathrm{C}$, com pontos quentes entre as chapas chegando a $30^{\circ} \mathrm{C}$. Na imagem 2, tem-se uma abertura na chapa do duto interno com região fria, indicando entrada falsa de ar. 
$\mathrm{Na}$ imagem 3 observa-se uma boca de visita fechada fora da posição, ocasionando também entrada falsa de ar para os gases de combustão. Já nas imagens 4, 5 e 6 existem pontos quentes devido a deformações nas chapas de alumínio, junções dos dutos e pontos de fixação dos suportes dos dutos, respectivamente. Locais estes favoráveis para a entrada de ar úmido para a região do isolante térmico, deteriorandoo e reduzindo sua eficiência na região afetada.

A validação dos pontos frios identificados como potenciais pontos de entrada falsa de ar para os gases, ocorreu através de uma confirmação presencial da sucção de ar frio em pontos de fácil acesso, tais como as bocas de visita e descontinuidades na base dos dutos internos.

\section{CONCLUSÃO}

Foram encontradas condições bastante favoráveis para a aplicação de termografia na identificação de pontos de entrada falsa de ar e vazamento de gases. Considerando que os dutos de gases após a caldeira possuem diferencial de pressão, negativa antes do precipitador e positiva após ele. Além do diferencial de temperatura, já que os gases se encontram em torno de $140^{\circ} \mathrm{C}$,

Foi concluído com este trabalho que a metodologia proposta é adequada para a identificação dos gradientes de temperatura em locais de difícil acesso, para o direcionamento dos pontos de reparo a serem executados nos dutos de gases de uma caldeira. Considerando que em torno de $25 \%$ do custo total de um shutdown de uma fábrica de celulose são gastos apenas com montagem e desmontagem de andaimes, tem-se um grande potencial em redução do custo operacional em uma parada de fábrica. Importante salientar que todos estes trabalhos de inspeção em locais de até 60 metros acima do solo, foram realizados sem a exposição de nenhum homem a altura acima de 2 metros.

Portanto, fica bastante evidente os ganhos substanciais quanto a qualidade da informação produzida, reduções de custo e tempo operacionais. Além da garantia da saúde e vida dos envolvidos.

\section{Agradecimentos}

Agradecemos a todos que participaram deste projeto, e em especial a empresa Suzano Papel e Celulose SA de Jacareí - SP, por acreditar e investir neste projeto piloto, e as empresas xd4solutions em parceria com a DS Engenharia Inovativa, executantes deste trabalho, por serem locomotivas que impulsionam 0 desenvolvimento tecnológico no Brasil. Agradeço também ao Programa de Pósgraduação em Engenharia Industrial da UFBA e a CAPES por fomentarem este artigo através do programa de doutorado. 


\section{REFERÊNCIAS}

${ }^{1}$ ALMEIDA, Gustavo M. A. Detecção de situações anormais em caldeiras de recuperação química. 2006. 21 f. Tese. Escola Politécnica da Universidade de São Paulo, São Paulo. 2006.

${ }^{2}$ OZAWA, Marcelo. Determinação experimental da resistividade ôhmica de cinzas volantes para projeto de precipitadores eletrostáticos. 2003. 61,62 f. Dissertação. Escola Politécnica da Universidade de São Paulo, São Paulo. 2003.

${ }^{3}$ ENTROP, A. G.; VASENEV, A. Infrared drones in the construction industry: designing a protocol for building thermography procedures. 11th Nordic Symposium on Building Physics, NSB2017. Trondheim, Norway, 2017.

${ }^{4}$ TAYLOR, T., COUNSELL, J., \& GILL, S. Energy efficiency is more than skin deep: Improving construction quality control in new-build housing using thermography. Energy and Buildings Jornal, 66, 222-231. United Kingdom. 2013.

${ }^{5}$ LUCCHI, E. Applications of the infrared thermography in the energy audit of buildings: A review. Renewable and Sustainable Energy Reviews Journal, 82, 30773090. 2018.

6 FLIR. Thermal imaging guidebook for building and renewable energy applications. Infrared Training Center (ITC). 2011

${ }^{7}$ DOSHVARPASSAND, S., WU, C., \& WANG, X. An overview of corrosion defect characterization using active infrared thermography. Infrared Physics and Technology, 96, 366-389. 2019. 